\title{
Sperm Quality as a Prognosticator in Intrauterine Insemination-treated Sub-fertile Saudi Patients AM Isa
}

\begin{abstract}
Background: Intrauterine insemination (IUI) is recognized as the most feasible infertility treatment, yet its success indications need further refinement.

Objective: This study aims to determine the most effective sperm parameters that help in prescribing IUI for infertility treatment within a Saudi population sample.

Method: We prospectively studied the inseminated post-wash total motile sperms count (TMSC), and the percentages of the fast(A)-plus-moderate(B)sperm progression of the specimens used for the IUI-treated infertility patients compared with the pregnancy rates (PR) corresponding to those parameters among a Saudi group of infertile patients.
\end{abstract}

Results: Our results showed clearly that over ten million TMSC would significantly make a better chance for conception after IUI treatment $(\rho=0.034)$. However, when the TMSC was sixty million or more, the chances for conception became significantly higher $(\rho=0.007)$. The A+B progression percentage did not significantly affect the PR $(\rho=0.295)$. The minimal post-wash TMSC that resulted in conception was 0.5 million sperms, with $\mathrm{A}+\mathrm{B}$ of $55 \%$, while the minimal post-wash $\mathrm{A}+\mathrm{B}$ progression percentage that resulted in conception was $25 \%$, with TMSC of eight million.

Conclusions: Although the semen quality of the studied population is declining, the TMSC is still the eligibility determining factor for IUI treated sub-fertile couples. A TMSC of at least ten million is still the recommended number for IUI suitability, although as few as 0.5 million still maintains the conception possibilities if the progression and morphology are normal.

Keywords: Infertility, insemination, pregnancy rate, sperm count

From: King Saud University, College of Medicine, Obstetrics and Gynaecology Department, Assisted Conception Unit, Riyadh, Saudi Arabia.

Correspondence: Dr M Isa, King Saud University, College of Medicine, OB GYNE Department, Assisted Conception Unit, Riyadh, Saudi Arabia. E-mail: issahmed@hotmail.com 


\section{INTRODUCTION}

The benefits of intrauterine insemination (IUI) were acknowledged early for infertility treatment with mild male factor, cervical factor, immunological factor, or unexplained reasons (1). Intrauterine insemination was also shown to be an expensive and less invasive procedure in comparison with the in vitro fertilization (IVF) procedure (2). Pregnancy rate (PR) difference after IUI is wide ranging because of the variations between the patient groups, and the variation of the studied parameters (3-7). However, results from some other studies indicate a degree of consistency (1).

The objective of this study is to evaluate the clinical results of IUI in infertile couples treated in our centre that belongs to a public hospital in Saudi Arabia. We wanted to determine which sperm parameters underly the success of IUI treatment in a local infertile population in comparison with other patient populations worldwide. We studied the relationship between the post-wash total motile sperms count, as well as the post-wash sum of fast-forward (A) and moderate-forward (B) sperm progression percentages and the corresponding PR of each as prognostic factors.

\section{SUBJECTS AND METHODS}

In this prospective cohort study of local sub-fertile patients who were eligible for IUI treatment, and over a period of twenty months (January 2010 to August 2011), we analyzed the cases and the outcome in terms of pregnancy rate, and compared that with results from previous studies around the world. 
For the purpose of accomplishing the goals of this study, cases with at least two million of initial TMSC and with $10 \%$ of $\mathrm{A}+\mathrm{B}$ progression were included, while cases with $100 \%$ of abnormal morphology were excluded. Also, couples with wives of 43 years or older (unless IUI treatment was their only option for personal reasons), or with blocked fallopian tubes were excluded.

After evaluating the infertility cases, those who approved their participation in the study signed informed consent. For those cases, two semen analyses were done; one semen specimen was washed to get an idea of the TMSC and the progression profile before the IUI procedure. Further tests for the male partners included serum levels of follicular stimulating hormone (FSH) and testosterone. For the female patients, the baseline hormonal levels of FSH, luteinizing hormone ( $\mathrm{LH})$, thyroid stimulating hormone (TSH), and prolactin were tested. Intrauterine insemination was then prescribed to those who were eligible and a gonadotropin stimulation protocol was assigned and patients were monitored by ultrasound for the proper timing of insemination. When the one/two leading follicle(s) reached at least $15 \mathrm{~mm}$ in diameter, instructions were given to inject $5000 \mathrm{IU}$ of human chorionic gonadotropin hormone $(\mathrm{HCH}) 36$ hours before the IUI time.

Semen specimens were centrifuged through density gradient (PureSperm 40/80 from Nidacon International, Sweden; Catalogue No. PSK-020) for 20 minutes, at $18000 \mathrm{rpm}$. The sperm pellet was then washed twice with HEPES-buffered media (Quinn's Advantage Medium, with HEPES, from SAGE Company; Cat. \# 1023), supplemented with serum (Quinn's Advantage Serum Protein Substitute SPS from SAGE Company; Cat. \# 3010). Final sperm pellet was constituted within $0.5 \mathrm{~mL}$ of the same media while all results were recorded, and data were analysed. 


\section{Statistical analysis}

SPSS software (Statistical Package for the Social Sciences, version 16.0, SPSS Inc., Chicago, Illinois, USA) was used for analysing the data. We used Chi-squared test to compare between pregnant and non-pregnant groups with respect to all variables. We assumed there was a significant difference when $\rho<0.05$.

\section{Ethical considerations}

The Institutional Review Board approved the above project under research project No. E-12-647. Complete informed written consents were obtained from all study participants ahead of all procedures. None of the authors has any kind of conflict of interest with any party related to any material used for this study.

\section{RESULTS}

\section{Post-wash total motile sperm count (TMSC) and pregnancy rate in IUI-treated patients}

Of the patients, $20.79 \%$ had TMSC of 5 million or less and this group had PR of $4.76 \%$ which was the lowest success rate amongst all groups (Table 1 ); $17.82 \%$ of the patients had $>5-10$ million, and resulted in a PR of $7.4 \%$. In total, $38.61 \%$ of the patients had ten millions $/ \mathrm{mL}$ or less of TMSC and they had an average PR of 5.98\%, which is significantly less compared to the rest of all the groups $(\rho=0.034)$. 
Table 1: Post-wash total motile sperm count (TMSC) and corresponding success rates of intrauterine insemination (IUI)-treated patients

\begin{tabular}{|c|c|c|c|c|}
\hline & Post-wash TMSC & Patients \# $n^{1}(\%)$ & Pregnancy rate & $\rho$-value \\
\hline 1 & $\leq 5$ million & $63(20.79 \%)$ & $3(4.76 \%)$ & \\
\hline 2 & $>5-10$ million & $54(17.82 \%)$ & $4(7.4 \%)$ & $\begin{array}{c}0.034 \text { (comparing PR of } \\
\leq 10 \mathrm{~m} \text { with }>10 \mathrm{~m}\end{array}$ \\
\hline 3 & $>10-20$ million & $59(19.47 \%)$ & $9(15.25 \%)$ & TMSC groups) \\
\hline 4 & $>20-30$ million & $55(18.15 \%)$ & $1(1.82 \%)$ & \\
\hline 5 & $>30-<60$ million & $50(16.5 \%)$ & $6(12 \%)$ & $\begin{array}{c}0.007 \text { (comparing PR of } \\
\geq 60 \mathrm{~m} \text { with }<60 \mathrm{~m}\end{array}$ \\
\hline 6 & $\geq 60$ million & $22(7.26 \%)$ & $6(27.27 \%)$ & TMSC groups) \\
\hline
\end{tabular}

Then there were $19.47 \%$ of the patients who had $>10-20$ million of TMSC, resulting in a relatively high success rate of $15.25 \%$. There was an unexpected drop in the PR to $1.82 \%$ in the $18.15 \%$ of the patients with $21-30$ million of TMSC. The PR increased to $12 \%$ with the following group of patients that constituted $16.5 \%$ of the total and who had $>30-59$ million of TMSC. The last group, $7.26 \%$ of the patients with TMSC of sixty million or more, resulted in the highly significant PR of $27.27 \%$ ( $\rho=0.007$ with the rest of all groups). 
Post-wash sperm fast-plus-moderate forward-progression $(A+B)$ percentages and its relation with the pregnancy rate in IUI-treated patients

Approximately $7 \%$ (6.71\%) of the total IUI-treated group had a sperm post-wash A+B\% of 35\% or less, and this group had a PR which was the lowest amongst all groups (Table 2). Additionally, $17.45 \%$ of patients had

Table 2: Post-wash fast forward and moderate forward progression and corresponding success rate of IUI-treated patients

\begin{tabular}{lllll}
\hline & A+B Progression \% & Patients \# and \% & Success Rate & P-value \\
\hline 1 & $\leq 35$ & $20(6.71 \%)$ & $1(5 \%)$ & \\
2 & $40-50$ & $52(17.45 \%)$ & $5(9.62 \%)$ & \\
3 & $55-65$ & $61(20.47 \%)$ & $8(13.11 \%)$ & 0.295 \\
4 & $70-80$ & $98(32.89 \%)$ & $7(7.14 \%)$ & \\
5 & $85-100$ & $67(22.48 \%)$ & $8(11.94 \%)$ & \\
\hline
\end{tabular}

a post-wash sperm of $40-50 \%$ of $\mathrm{A}+\mathrm{B}$ progression types, and this group resulted in a PR of 9.62\%. One-fifth $(20.47 \%)$ of the patients had A+B progression profile of $55-65 \%$, with a PR of $13.11 \%$, which was the highest amongst all the groups. One-third $(32.89 \%)$ of the total treated group had $70-80 \%$ of $\mathrm{A}+\mathrm{B}$ progression types, resulting in a PR of $7.14 \%$. The final group comprised $22.48 \%$ of the all patients, and this group had $80-100 \%$ of $\mathrm{A}+\mathrm{B}$ progression and resulted in a PR of $11.94 \%$. There was no significant difference amongst all the groups. 


\section{DISCUSSION}

Our results proved that relatively low TMSC for IUI was still hopeful. A PR of $4.76 \%$ was obtained when the post-wash TMSC was five million or less. This finding is in contrast with those of Khalil et al (8), and Yang et al (9), who considered that TMSC of five million or more was the least to consider for IUI treatment. Wainer et al (10) also considered TMSC of five million as the critical number only when the normal sperm morphology was less than $30 \%$. In fact, one of our cases that resulted in pregnancy had only 0.5 million motile sperms (with 55\% A+B sperm progression and 19\% normal morphology) and another successful case had only one million motile sperms though the $\mathrm{A}+\mathrm{B}$ progression was only $25 \%$ and $12 \%$ normal morphology. These findings fine-tune the way we think about the minimal post-wash TMSC that would be good enough for IUI treatment, though the success rate is expectedly low. It was many years ago that Campana et al (11) and Ombelet et al (12) reported similar results to our findings. They stated that IUI success rate was significantly adversely affected at values only less than one million. Pasqualotto et al (13) also reported a reasonable $3.5 \%$ live birth rate at TMSC of 0.5 million.

In our study, the second group (17.82\% of the patients) was inseminated with TMSC of $\geq$ 5-10 million, with PR of 7.4\%, which is still encouraging, especially for cases that refuse regular IVF.

The third category comprised $19.47 \%$ of the patients, and was inseminated with $>10-20$ million, and had a PR of $15.25 \%$ which is significantly higher than the previous groups. We currently try processing two semen specimens for patients with low sperm count so that the final TMSC would fall into this category. The results are promising and more data are being collected. 
The fourth group of $18.15 \%$ of the patients was inseminated with $>20-30$ million TMSC and had a PR of only $1.82 \%$, which is very low compared to all the studied categories. It was found that a high percentage of the patients in this group were diagnosed with hormonal imbalance such as endometriosis, high FSH, and hyperprolactinaemia that caused their infertility and impacted the IUI treatment results. These findings are in line with Burwinkel et al (14), and Rajashekar et al (15).

The pregnancy rate rose again to $12 \%$ with the fifth group (16.5\% of the patients) that was inseminated with TMSC of $>30-59$ million. These results are comparable with that of Demir et al (16). Also, Doripurev et al (17) concluded that the PR per cycle was significantly higher $(8.2 \%)$ with TMSC of at least 10 million.

Pregnancy rate significantly improved as it reached $27.27 \%$ in the sixth group, when the TMSC was $\geq 60, \rho=0.007$ compared with all other groups. This result is comparable to IVF results in some cases, which endorses that TMSC plays the most important role. The reason such good quality sperm did not result in natural pregnancy is indicated by Alkhayali et al (18) who noted that sperm chromatin integrity may be abnormal in men enrolled in IUI treatment cycles, despite the fact that most of these men look normozoospermic. There could also be other hidden reasons that affect the success rate.

This variation in results is contrary with what Pasqualotto et al (13) has reported. They stated that what mattered only was the post-wash sperm-motility percentage. On the other hand, Zadehmodarres et al (19) reported that the only determining factor for IUI success was that the final fast progressing sperm concentration after preparation was $\geq 1 \times 10^{6} / \mathrm{mL}$. In the survey done by Rawal et al (20) on 150 reproductive centres in the United Kingdom, the TMSC factor was the number three factor after progression and morphology. 
We also found that the cut-off percentage of $\mathrm{A}+\mathrm{B}$ progression, below which the PR noticeably dropped, was $40 \%$ (Table 2). Pregnancy rate of $5 \%$ was the result when $\mathrm{A}+\mathrm{B}$ progression ranged between $10 \%$ and $35 \%$. The lowest percentage that resulted in pregnancy was $25 \%$, with eight million TMSC. This could be around the value of $10 \%$ of fast progressive sperms described by Haim et al (21).

For the rest of the $\mathrm{A}+\mathrm{B}$ progression ranges, $(40-50 \%, 55-65 \%, 70-80 \%$, and $85-100 \%)$ the PRs were $9.62 \%, 13.11 \%, 7.14 \%$, and $11.94 \%$, respectively, $(\rho=0.295)$. Once the A+B progression rates are over $25 \%$, the success expectations become higher. When Zhao et al (22) considered that $\mathrm{A}+\mathrm{B}$ progression was detrimental for IUI success, they may have meant the critical minimal value of it. However, these findings are neither in complete agreement with Shulman et al (23) who indicated that good sperm progressive motility was the only sperm indicator for IUI success, nor do they agree with Zadehmodarres et al (19) who stated that the only determining factor for IUI success was that the post sperm preparation fast progression count had to be $\geq 1 \times 10^{6} / \mathrm{mL}$.

In regards to the sperm morphology, no difference was detected between the group that resulted in pregnancy with normal morphology of $8-28 \%$, and the unsuccessful group of normal morphology of $8-21 \%$. That was probably because both ranges are higher than the $4 \%$ threshold value for successful pregnancy in vivo as stated by many authors and reported by Van Waart et al. (24), which nullified the morphology effect in this study.

The overall results of the study are important, especially since a higher percentage of sperm abnormalities was detected in males of infertile couples in Saudi Arabia compared with other regions in the world (25). 


\section{CONCLUSION}

Although the semen quality of the studied population is declining, the TMSC is still the eligibility determining factor for IUI treated sub-fertile couples. A TMSC of at least ten million is still the recommended number for IUI suitability, although as few as 0.5 million still maintains the conception possibilities if the progression and morphology are normal

\section{ACKNOWLEDGEMENTS}

The author would like to thank the College of Medicine Research Centre, the Deanship of Scientific Research, Kind Saud University, for providing all the support for this work, the author also would like to greatly thank the Andrology Laboratory team for their collecting the data used for the study. 


\section{REFERENCES}

1. Ibérico G, Vioque J, Ariza N, Lozano JM, Roca M, Llácer J et al. Analysis of factors influencing pregnancy rates in homologous intrauterine insemination. Fertil Strel 2004; 81:1308-13.

2. Kossakowski J, Stephenson M, Smith H. Intrauterine insemination with husband's sperm: comparison of pregnancy rates in couples with cervical factor, male factor, immunological factor and idiopathic infertility. Aust N Z J Obstet Gynaecol 1993; 33: $183-6$.

3. Allen NC, Herbert CM 3rd, Maxson WS, Rogers BJ, Diamond MP, Wentz AC. Intrauterine insemination: a critical review. Fertil Steril 1985; 44: 569-80.

4. Nuojua-Huttunen S, Tomas C, Bloigu R, Tuomivaara L, Martikainen H. Intrauterine insemination treatment in subfertility: an analysis of factors affecting outcome. Hum Reprod 1999; 14: 698-703.

5. Gezginç K, Görkemli H, Celik C, Karatayli R, Ciçek MN, Olakoglu MC. Comparison of single versus double intrauterine insemination. Taiwan J Obstet Gynecol 2008; 7: 57-61.

6. Demirol A, Gurgan T. Comparison of different gonadotrophin preparations in intrauterine insemination cycles for the treatment of unexplained infertility: a prospective, randomized study. Hum Reprod 2007; 22: 97-100.

7. Freour T, Jean M, Mirallie S, Langlois ML, Dubourdieu S, Barriere P. Predictive value of CASA parameters in IUI with frozen donor sperm. Int J Androl 2009; 32: 498-504.

8. Khalil MR, Rasmussen PE, Erb K, Laursen SB, Rex S, Westergaard LG. Homologous intrauterine insemination. An evaluation of prognostic factors based on a review of 2473 cycles. Acta Obstet Gynecol Scand 2001; 80: 74-81. 
9. Yang JH, Wu MY, Chao KH, Chen SU, Ho HN, Yang YS. Controlled ovarian hyperstimulation and intrauterine insemination in subfertility. How many treatment cycles are sufficient? J Reprod Med 1998; 43: 903-8.

10. Wainer R, Albert M, Dorion A, Bailly M, Bergère M, Lombroso R et al. Influence of the number of motile spermatozoa inseminated and of their morphology on the success of intrauterine insemination. Hum Reprod 2004; 19: 2060-5.

11. Campana A, Sakkas D, Stalberg A, Bianchi PG, Comte I, Pache T et al. Intrauterine insemination: evaluation of the results according to the woman's age, sperm quality, total sperm count per insemination and life table analysis. Hum Reprod 1996; 11: 732-6.

12. Ombelet W, Deblaere K, Bosmans E, Cox A, Jacobs P, Janssen M et al. Semen quality and intrauterine insemination. Reprod Biomed Online 2003; 7: 485-92.

13. Pasqualotto EB, Daitch JA, Hendin BN, Falcone T, Thomas AJ Jr, Nelson DR et al Relationship of total motile sperm count and percentage motile sperm to successful pregnancy rates following intrauterine insemination. J Assist Reprod Genet 1999; 16: $476-82$.

14. Burwinkel TH, Buster JE, Scoggan JL, Carson SA. Basal follicle stimulating hormone (FSH) predicts response to controlled ovarian hyperstimulation $(\mathrm{COH})$-intrauterine insemination (IUI) therapy. J Assist Reprod Genet 1994; 11: 24-7.

15. Rajashekar L, Krishna D, Patil M. Polycystic ovaries and infertility: our experience. J Hum Reprod Sci 2008; 1: 65-72.

16. Demir B, Dilbaz B, Cinar O, Karadag B, Tasci Y, Kocak M et al. Factors affecting pregnancy outcome of intrauterine insemination cycles in couples with favourable female characteristics. J Obstet Gynaecol 2011; 31: 420-3. 
17. Dorjpurev U, Kuwahara A, Yano Y, Taniguchi T, Yamamoto Y, Suto A et al. Effect of semen characteristics on pregnancy rate following intrauterine insemination. $\mathrm{J}$ Med Invest $2011 ; \mathbf{5 8 :} 127-33$.

18. Alkhayal A, San Gabriel M, Zeidan K, Alrabeeah K, Noel D, McGraw R et al. Sperm DNA and chromatin integrity in semen samples used for intrauterine insemination. J Assist Reprod Genet 2013; 30: 1519-24.

19. Zadehmodarres S, Oladi B, Saeedi S, Jahed F, Ashraf H. Intrauterine insemination with husband semen: an evaluation of pregnancy rate and factors affecting outcome. $\mathrm{J}$ Assist Reprod Genet 2009; 26: 7-11.

20. Rawal N, Drakeley A, Haddad N. Intrauterine insemination practice in the UK. J Obstet Gynaecol 2008; 28: 738-41.

21. Haim D, Leniaud L, Porcher R, Martin-Pont B, Wolf JP, Sifer C et al. Prospective evaluation of the impact of sperm characteristics on the outcome of intra-uterine insemination. Gynecol Obstet Fertil 2009; 37: 229-35.

22. Zhao Y, Vlahos N, Wyncott D, Petrella C, Garcia J, Zacur H et al. Impact of semen characteristics on the success of intrauterine insemination. J Assist Reprod Gene 2004; 21: $143-8$.

23. Shulman A, Hauser R, Lipitz S, Frenkel Y, Dor J, Bider D et al. Sperm motility is a major determinant of pregnancy outcome following intrauterine insemination. J Assist Reprod Genet 1998; 15: 381-5.

24. Van Waart J, Kruger TF, Lombard CJ, Ombelet W. Predictive value of normal sperm morphology in intrauterine insemination (IUI): a structured literature review. Hum Reprod Update 2001; 7: 495-500. 
25. AlEnezi H, Isa AM, Abu-Rafea B, Madbouly K, Binsaleh S. Pattern of semen fluid abnormalities in male partners of infertile couples in Riyadh, Saudi Arabia. Can J Urol 2014; 21: 7322-5. 